\title{
Sound field separation with sound pressure and particle velocity measurements
}

\author{
Fernandez Grande, Efren; Jacobsen, Finn; Leclère, Quentin
}

Published in:

Acoustical Society of America. Journal

Link to article, DOI:

$10.1121 / 1.4763988$

Publication date:

2012

Document Version

Publisher's PDF, also known as Version of record

Link back to DTU Orbit

Citation (APA):

Fernandez Grande, E., Jacobsen, F., \& Leclère, Q. (2012). Sound field separation with sound pressure and particle velocity measurements. Acoustical Society of America. Journal, 132(6), 3818-3825.

https://doi.org/10.1121/1.4763988

\section{General rights}

Copyright and moral rights for the publications made accessible in the public portal are retained by the authors and/or other copyright owners and it is a condition of accessing publications that users recognise and abide by the legal requirements associated with these rights.

- Users may download and print one copy of any publication from the public portal for the purpose of private study or research.

- You may not further distribute the material or use it for any profit-making activity or commercial gain

- You may freely distribute the URL identifying the publication in the public portal

If you believe that this document breaches copyright please contact us providing details, and we will remove access to the work immediately and investigate your claim 


\title{
Sound field separation with sound pressure and particle velocity measurements
}

\author{
Efren Fernandez-Grande ${ }^{\text {a) }}$ and Finn Jacobsen \\ Acoustic Technology, Department of Electrical Engineering, DTU Technical University of Denmark, \\ Building 352, Oersteds Plads, DK-2800 Kongens Lyngby, Denmark \\ Quentin Leclère \\ Laboratoire Vibrations Acoustique, INSA Lyon, F-69621 Villeurbanne Cedex, France
}

(Received 10 May 2012; revised 26 September 2012; accepted 3 October 2012)

\begin{abstract}
In conventional near-field acoustic holography (NAH) it is not possible to distinguish between sound from the two sides of the array, thus, it is a requirement that all the sources are confined to only one side and radiate into a free field. When this requirement cannot be fulfilled, sound field separation techniques make it possible to distinguish between outgoing and incoming waves from the two sides, and thus NAH can be applied. In this paper, a separation method based on the measurement of the particle velocity in two layers and another method based on the measurement of the pressure and the velocity in a single layer are proposed. The two methods use an equivalent source formulation with separate transfer matrices for the outgoing and incoming waves, so that the sound from the two sides of the array can be modeled independently. A weighting scheme is proposed to account for the distance between the equivalent sources and measurement surfaces and for the difference in magnitude between pressure and velocity. Experimental and numerical studies have been conducted to examine the methods. The double layer velocity method seems to be more robust to noise and flanking sound than the combined pressure-velocity method, although it requires an additional measurement surface. On the whole, the separation methods can be useful when the disturbance of the incoming field is significant. Otherwise the direct reconstruction is more accurate and straightforward.
\end{abstract}

(C) 2012 Acoustical Society of America. [http://dx.doi.org/10.1121/1.4763988]

PACS number(s): 43.60.Sx, 43.60.Pt, 43.20.Rz [EJS]

Pages: $3818-3825$

\section{INTRODUCTION}

Near-field acoustic holography (NAH) $)^{1,2}$ is a well established sound source identification technique that makes use of near-field measurements in order to reconstruct and visualize the complete sound field radiated by a sound source, i.e., sound pressure, particle velocity, and sound intensity, over a three-dimensional space near the source. In conventional NAH, it is not possible to distinguish between sound coming from the two sides of the array. Therefore, a freefield half-space is required where all the sound sources are confined to only one side.

If there are mutually incoherent sources on the two sides of the array, it is possible to separate their contribution based on their statistical properties, ${ }^{3-6}$ or if only one source is of interest and its phase reference is available, the "disturbing" sound can be simply averaged out. However, if the sound from the two sides of the array is due to coherent sources, it is not possible to make use of their statistical properties for the separation. In this case, sound field separation methods, which make use of directional information to estimate the propagation direction of the waves, can be very useful.

The first separation methods, some of which were proposed more than two decades ago, ${ }^{7-11}$ rely on measurements

\footnotetext{
a) Author to whom correspondence should be addressed. Electronic mail: efg@elektro.dtu.dk
}

of the sound pressure in two closely spaced parallel planes. In recent years several separation methods based on the combined measurement of the sound pressure and the particle velocity have appeared. ${ }^{12-15}$ More recently, a method was presented that made use of particle velocity measurements in two closely spaced parallel planes. ${ }^{16}$

Two new methods are proposed in this paper, one that relies on measurement of the particle velocity in two layers $(\mathrm{u}-\mathrm{u})$, and another that relies on measurement of the sound pressure and particle velocity in a single layer $(\mathrm{p}-\mathrm{u})$. The present study differs from previous ones (Ref. 16) in that it examines the $\mathrm{u}-\mathrm{u}$ measurement principle in a general sense, considering the separation of both the sound pressure and the particle velocity fields, and is based on the equivalent source method, ${ }^{17,18}$ thus it can be applied to arbitrarily shaped sources. Furthermore, the proposed methods ( $\mathrm{u}-\mathrm{u}$ and $\mathrm{p}-\mathrm{u}$ ) use independent transfer matrices for the outgoing and incoming waves, and an optional weighting to compensate for the distance between the equivalent sources and the measurement surface. The proposed p-u method is based on a weighted least squares inversion that compensates for the difference in magnitude between pressure and velocity.

One of the main potentials of separation techniques is the possibility of using NAH in non-anechoic environments such as conventional rooms or other enclosed spaces, where a source may be radiating in the presence of multiple reflections. The performance of double layer 
pressure techniques in such enclosed spaces has been addressed previously. ${ }^{19-21}$ On the contrary, the recent separation methods based on pressure and velocity measurements have mostly been examined with a single disturbing source ${ }^{12-14,16}$ or a single reflection, ${ }^{15}$ but not for the case where multiple reflections from different directions occur. In fact, the measurement of the normal component of the particle velocity can be favorable in this case, because the influence of reflected waves arriving from the edges of the measurement aperture is naturally suppressed. The separation methods proposed in this study are examined for the case where a sound source is radiating into an enclosed space in the presence of multiple reflections.

\section{THEORY}

\section{A. Double layer particle velocity}

Given a sound field consisting of outgoing and incoming waves, the normal component of the particle velocity in two layers, $\mathbf{r}_{h 1}$ and $\mathbf{r}_{h 2}$, can be expressed as the result of the superposition of the sound field produced by a distribution of point sources at the two sides of the measurement aperture (see Fig. 1). These so-called equivalent sources are distributed over the surfaces $\mathbf{r}_{a}$ and $\mathbf{r}_{b}$, thus,

$$
\begin{aligned}
u_{n}\left(\mathbf{r}_{h 1}\right)= & -\sum_{k}^{N} q_{(1, k)} G_{u}\left(\mathbf{r}_{h 1}, \mathbf{r}_{a k}\right) \\
& -\sum_{k}^{M} q_{(2, k)} G_{u}\left(\mathbf{r}_{h 1}, \mathbf{r}_{b k}\right),
\end{aligned}
$$

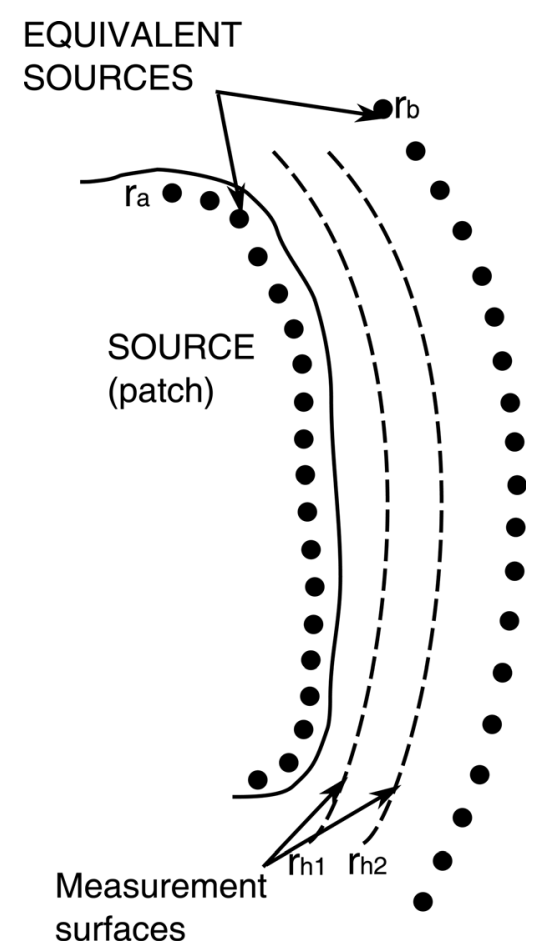

FIG. 1. Diagram of the double layer equivalent source method (for a patch of the source).

$$
\begin{aligned}
u_{n}\left(\mathbf{r}_{h 2}\right)= & -\sum_{k}^{N} q_{(1, k)} G_{u}\left(\mathbf{r}_{h 2}, \mathbf{r}_{a k}\right) \\
& -\sum_{k}^{M} q_{(2, k)} G_{u}\left(\mathbf{r}_{h 2}, \mathbf{r}_{b k}\right),
\end{aligned}
$$

where $q_{k}$ is the strength of each equivalent source and the function $G_{u}$ is the derivative in the normal direction (to the equivalent source surface) of the Green's function in freespace,

$$
\begin{aligned}
& G_{u}\left(\mathbf{r}, \mathbf{r}_{0}\right)=\frac{\partial}{\partial n} G\left(\mathbf{r}, \mathbf{r}_{0}\right), \\
& G\left(\mathbf{r}, \mathbf{r}_{0}\right)=\frac{e^{-j k\left|\mathbf{r}-\mathbf{r}_{0}\right|}}{4 \pi\left|\mathbf{r}-\mathbf{r}_{0}\right|}
\end{aligned}
$$

Note that the time dependence $e^{j \omega t}$ has been omitted.

Equations (1) and (2) can be expressed in matrix form as

$$
\left[\begin{array}{l}
\mathbf{u}_{h 1} \\
\mathbf{u}_{h 2}
\end{array}\right]=-\left[\begin{array}{ll}
\mathbf{G}_{a \mid h 1}^{u} & \mathbf{G}_{b \mid h 1}^{u} \\
\mathbf{G}_{a \mid h 2}^{u} & \mathbf{G}_{b \mid h 2}^{u}
\end{array}\right] \cdot\left[\begin{array}{l}
\mathbf{q}_{1} \\
\mathbf{q}_{2}
\end{array}\right] .
$$

From Eq. (5), the strength of the equivalent sources $\mathbf{q}_{1}$ and $\mathbf{q}_{2}$ can be estimated from the measured velocities by means of a regularized inversion ${ }^{22}$ of the matrix. Then, the outgoing and incoming sound can be estimated via the Green's function between the equivalent sources and the reconstruction positions as

$$
\begin{aligned}
& \mathbf{u}_{s}^{(o)}=-\mathbf{G}_{a \mid s}^{u} \mathbf{q}_{1}, \\
& \mathbf{p}_{s}^{(o)}=j \omega \rho \cdot \mathbf{G}_{a \mid s} \mathbf{q}_{1}, \\
& \mathbf{u}_{s}^{(i)}=-\mathbf{G}_{b \mid s}^{u} \mathbf{q}_{2}, \\
& \mathbf{p}_{s}^{(i)}=j \omega \rho \cdot \mathbf{G}_{b \mid s} \mathbf{q}_{2} .
\end{aligned}
$$

The superscripts $(o)$ and $(i)$ denote the outgoing and incoming fields, respectively, and the subscript s the reconstruction positions.

Note that this method, because of being based on an equivalent source model, is not limited to separable geometries but can handle arbitrarily shaped sources.

\section{B. Single layer pressure-velocity}

Sound arriving from the two sides of the array can also be separated based on the combined measurement of sound pressure and particle velocity. ${ }^{12-15}$ In the present study, the separation is based on the equivalent source method with independent transfer matrices for the outgoing and incoming sound.

Based on the measured sound pressure and particle velocity,

$$
\left[\begin{array}{l}
\mathbf{p}_{h} \\
\mathbf{u}_{h}
\end{array}\right]=\left[\begin{array}{cc}
j \omega \rho \mathbf{G}_{a \mid h} & j \omega \rho \mathbf{G}_{b \mid h} \\
-\mathbf{G}_{a \mid h}^{u} & -\mathbf{G}_{b \mid h}^{u}
\end{array}\right] \cdot\left[\begin{array}{l}
\mathbf{q}_{1} \\
\mathbf{q}_{2}
\end{array}\right],
$$


from which $\mathbf{q}_{1}$ and $\mathbf{q}_{2}$ can be estimated by means of a regularized inversion, and the outgoing and incoming sound pressure and particle velocity can be reconstructed using Eqs. (6)-(9).

However, if this system was solved as a conventional least squares problem, the weight of the velocity field in the solution would be less than that of the pressure, because the former is typically of much smaller magnitude [by approximately $\rho c$, as follows from Euler's equation of motion $\mathbf{u}=-\nabla p /(j \omega \rho)]$, Thus the minimization of the residual would depend very strongly on the pressure vector. It is more appropriate to solve the system by means of a weighted least squares solution. ${ }^{23}$ The solution for the vector $\mathbf{q}$ in this case is

$$
\mathbf{q}=\left(\mathbf{W G}_{h}\right)^{+} \mathbf{W} \mathbf{b},
$$

where $\mathbf{G}_{h}$ is the transfer matrix as in Eq. (10), $\mathbf{b}$ is the column vector with the measured pressure and velocity, and $\mathbf{W}$ is the weighting diagonal matrix. The superscript + denotes the regularized pseudo-inverse:

$$
\left(\mathbf{W G}_{h}\right)^{+}=\left(\left[\mathbf{W G}_{\mathbf{h}}\right]^{H} \mathbf{W} \mathbf{G}_{\mathbf{h}}+\lambda \mathbf{I}\right)^{-1}\left[\mathbf{W G}_{\mathbf{h}}\right]^{H},
$$

where $\lambda$ is the Tikhonov regularization parameter. Note that regularization is essential when the solution is backpropagated.

A straightforward and robust choice for the weighting matrix is to divide the pressures with the norm of all the pressure inputs, and the velocities with the norm of all velocity inputs. Thus the weighting matrix is diagonal with the inverse of the pressure and velocity norms, $[\mathbf{W}]_{2 m \times 2 m}$ $=\operatorname{diag}\left([1 /\|\mathbf{p}\|]_{1 \times m},[1 / \| \mathbf{u}||]_{1 \times m}\right)$.

The purpose of this weighting is to equalize the influence of the measured inputs to obtain a meaningful solution, equally based on the pressure and particle velocity measurements. This weighting reduces the condition number of the transfer matrix considerably. Thus, the obtained solution is much less sensitive to noise and is more robust. The results obtained with this methodology are similar to the results obtained by inverting the sound pressure and velocity separately, although in this case a single inversion is required.

Apart from the inversion, the method described here differs from the one in Ref. 14 in that the equivalent sources of the outgoing and incoming fields, $\mathbf{G}_{a \mid h}$ and $\mathbf{G}_{b \mid h}$, can be placed asymmetrically, so that they can model the sound from the two sides independently. Thus, if the incoming sound is radiated by a source that is not placed equidistantly from the array, the equivalent sources from one side can be retracted accordingly, and the results can be improved. This property is also useful when the method is applied to sources with arbitrary geometries because the distribution of equivalent sources at the two sides can be modified according to the source geometries.

It should be noted that if the retraction distance of the equivalent sources (relative to the reconstruction surface) at the two sides of the array is significantly different, an additional right-hand weighting would be applied to the transfer matrix, to guarantee that all equivalent sources have the same weight in the minimization of the regularized solution norm, regardless of the distance to the measurement surface. The system to be solved in this case would be

$$
[\mathbf{W b}]=\left[\mathbf{W G}_{\mathbf{h}} \mathbf{M}\right] \mathbf{q}_{\mathbf{M}}
$$

where $\mathbf{M}$ is the new weighting diagonal matrix, and $\mathbf{q}_{\mathbf{M}}=\mathbf{M}^{-1} \mathbf{q}$. An appropriate weighting choice for the matrix $\mathbf{M}$ is the distance from each equivalent source to the measurement surface. ${ }^{24}$ In this way, no excessive energy is attributed to the equivalent sources that are closer to the measurement positions. However, this weighting is not necessary in the present study.

\section{NUMERICAL STUDY}

A numerical study has been conducted to examine the methods described in the foregoing. The source used for the experiment was a simply supported baffled steel plate of $30 \times 30 \mathrm{~cm}^{2}, 1 \mathrm{~mm}$ thick, driven at the center by a point force of $0.1 \mathrm{~N}$. The pressure and velocity radiated by the plate were calculated by numerically evaluating the Rayleigh integral using a discrete grid of $35 \times 35$ positions. The measurement grid consisted of $11 \times 11$ uniformly spaced positions over an area of $40 \times 40 \mathrm{~cm}^{2}$, with $4 \mathrm{~cm}$ inter-spacing distance. Normally distributed background noise of $30 \mathrm{~dB}$ signal-to-noise ratio was added to the simulated measurements.

The normalized error in $\mathrm{dB}$ between the "true" free field radiation by the plate and the one reconstructed with the different techniques, was calculated as

$$
\begin{aligned}
& E_{p}[\mathrm{~dB}]=20 \log _{10}\left(\frac{\left\|\mathbf{p}_{\text {plate }}-\mathbf{p}_{s}\right\|_{2}}{\left\|\mathbf{p}_{\text {plate }}\right\|_{2}}\right), \\
& E_{u}[\mathrm{~dB}]=20 \log _{10}\left(\frac{\left\|\mathbf{u}_{\text {plate }}-\mathbf{u}_{s}\right\|_{2}}{\left\|\mathbf{u}_{\text {plate }}\right\|_{2}}\right),
\end{aligned}
$$

where $\mathbf{p}_{\text {plate }}$ and $\mathbf{u}_{\text {plate }}$ are the free field pressure and normal velocity radiated by the plate, and $\mathbf{p}_{s}$ and $\mathbf{u}_{s}$ are the reconstructed ones with each of the methods.

\section{A. Plate disturbed by an incident plane wave}

In order to study how incoming sound influences the reconstruction, the case of a baffled vibrating plate radiating sound in the presence of an incoming plane wave is considered. The back-scattering from the source is modeled by means of a reflected plane wave. The measurement planes are $z_{h 1}=7 \mathrm{~cm}$ and $z_{h 2}=12 \mathrm{~cm}$ for the $\mathrm{u}-\mathrm{u}$ method, and $z_{h 1}=7 \mathrm{~cm}$ for the $\mathrm{p}-\mathrm{u}$ method. The reconstruction plane is also $z_{h 1}$. The equivalent sources are retracted two interspacing distances from the reconstruction planes.

Figure 2 shows the reconstruction error at $z_{h 1}$ of the sound pressure and normal velocity as a function of frequency when there is an incoming plane wave with a $45^{\circ}$ elevation. The lower part of the figure shows the ratio in $\mathrm{dB}$ between the magnitude of the plate's free-field radiation and the incoming plus back-scattered plane waves (at $z_{h 1}$ for 



Free-field to disturbance ratio

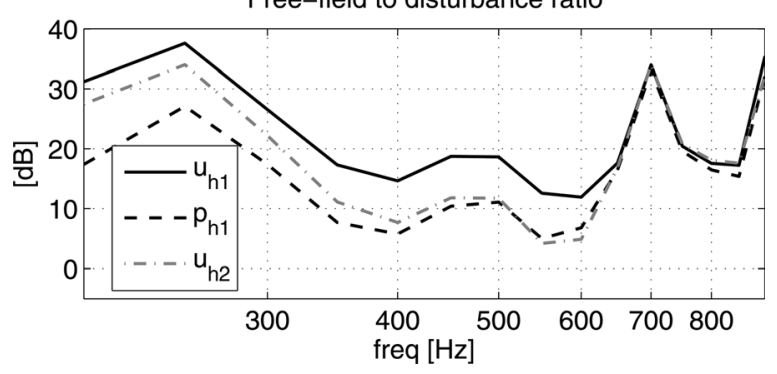

FIG. 2. Top: Reconstruction error of the field radiated by a baffled plate in the presence of an incident plane wave coming from $45^{\circ}$ elevation as a function of frequency. Bottom: Ratio between the radiated sound by the plate and the incident plus backscattered plane waves at $z_{h 1}$ and $z_{h 2}$.

pressure and velocity, and at $z_{h 2}$ for second layer of the $\mathrm{u}-\mathrm{u}$ measurement).

In the low frequency range (below $350 \mathrm{~Hz}$ ) the velocity based methods, "u" and "u-u," are consistently the most accurate due to the lesser disturbance of the velocity field at the source's boundary (due to the mutual canceling of incoming and scattered waves). Above $500 \mathrm{~Hz}$, the disturbance of the pressure at $z_{h 1}$ is less critical than that of the velocity at $z_{h 2}$, and consequently the results with the p-u method are slightly better (the disturbance of the pressure and velocity fields is illustrated in Fig. 2-bottom).

On the whole, the accuracy of the methods depends strongly on if the incoming and the back-scattered waves interfere constructively or destructively and the resulting disturbance of the sound radiated by the plate. This explains the accuracy of the $\mathrm{p}-\mathrm{u}$ and $\mathrm{u}-\mathrm{u}$ separation methods as a function of frequency (e.g., if the stand-off distance corresponds roughly to $\lambda_{z} / 4$, the incident and back-scattered pressures cancel each other). Additionally, it should be noted that the u-u method has a larger stand-off distance due to the double-layer configuration, and consequently a larger back-propagation to the reconstruction surface.

Figure 3 shows the reconstruction error as a function of the angle of incidence of the plane wave. The angle of incidence varies from $\theta=90^{\circ}$, where the wave is propagating perpendicular to the normal direction of the plate $\left(k_{z}=0\right)$, to $\theta=0^{\circ}$ where it is propagating towards the plate $\left(k_{z}=k\right)$. When the incident plane wave travels tangentially to the plate $\left(\theta=90^{\circ}\right)$ the normal component of the particle velocity is undisturbed. This explains the low reconstruction error of the velocity based methods between $90^{\circ} \geq \theta \geq 60^{\circ}$.

\section{B. Plate radiating into an enclosed space}

A preliminary numerical experiment with the baffled plate radiating into a rectangular room was considered. The room is of dimensions $3 \times 2.5 \times 2 \mathrm{~m}^{3}$, and the plate is baffled in the center of the $2.5 \times 3 \mathrm{~m}^{2}$ wall. In this numerical experiment, only the first reflection from each wall and the backscattering from the source were taken into account. The
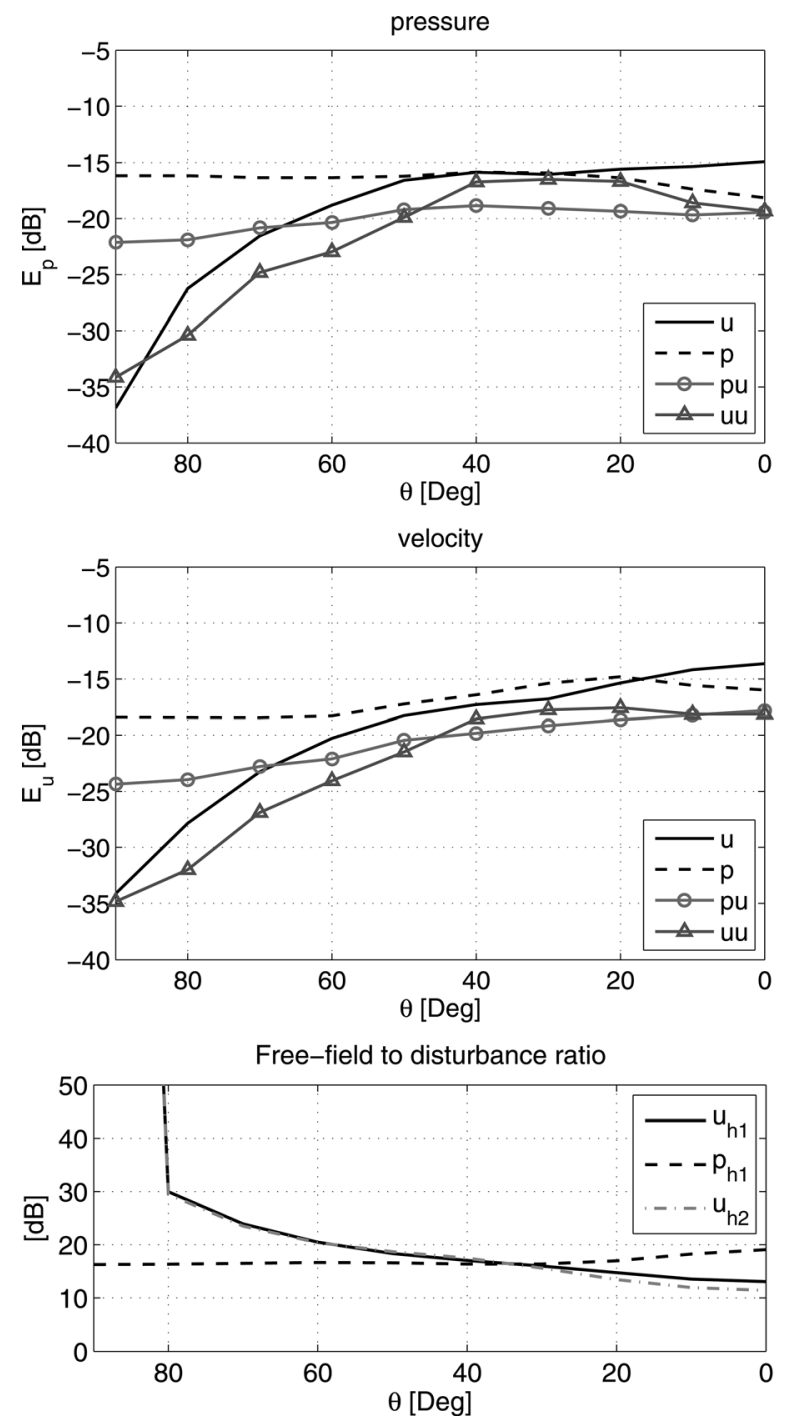

FIG. 3. Top: Reconstruction error of the field radiated by a baffled plate in the presence of an incident plane wave coming from the opposite side as a function of the incidence angle ( $\theta=0$ corresponds to frontal incidence). Bottom: Ratio between the radiated sound by the plate and the incident plus backscattered plane waves $z_{h 1}$ and $z_{h 2}(f=800 \mathrm{~Hz})$. 
measurement planes were $z_{h 1}=10 \mathrm{~cm}$ and $z_{h 2}=15 \mathrm{~cm}$, and the reconstruction plane was also $z_{h 1}$ (see Fig. 4). The equivalent sources were retracted two inter-spacing distances from the reconstruction planes.

Figure 5 shows the error of the reconstructions compared to the free field radiation from the plate. At low frequencies (below $400 \mathrm{~Hz}$ ) the velocity based methods are the most accurate due to the lesser disturbance of the normal velocity at the boundary of the source, whereas at higher frequencies the separation methods ( $\mathrm{p}-\mathrm{u}$ and $\mathrm{u}-\mathrm{u}$ ) provide on the whole a better estimation (unless the disturbance is small, in which case the direct reconstruction is better, e.g., pressure at $800 \mathrm{~Hz}$ ). The results indicate once again that the accuracy of the reconstruction depends significantly on the magnitude of the disturbing sound due to reflections.

Figure 6 shows the condition numbers of the matrices used by the separation methods to relate the measured field to the strength of the equivalent sources [see Eqs. (5) and (10)], as well as of the matrices of the direct reconstructions (note that the p-u method is shown for the conventional least squares and the proposed weighted least squares solution). The condition number is an indication of how sensitive a method is to measurement noise, and how sensitive the solution is to small changes in the input data. It can be seen that the condition number of the weighted least squares solution is substantially lower (it is still higher than the other methods due to the intrinsic differences between the pressure and velocity propagators). These results indicate that the velocity based methods, single or double layer, are considerably more robust to measurement noise than the pressure or pressure-velocity ones.

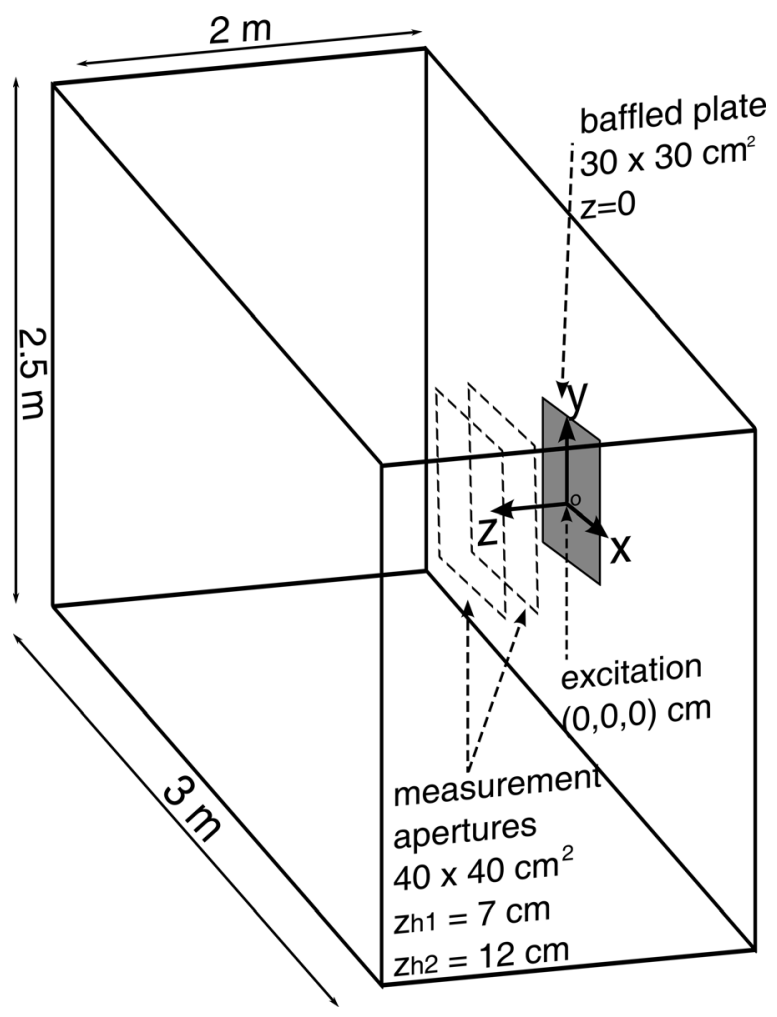

FIG. 4. Numerical set-up.
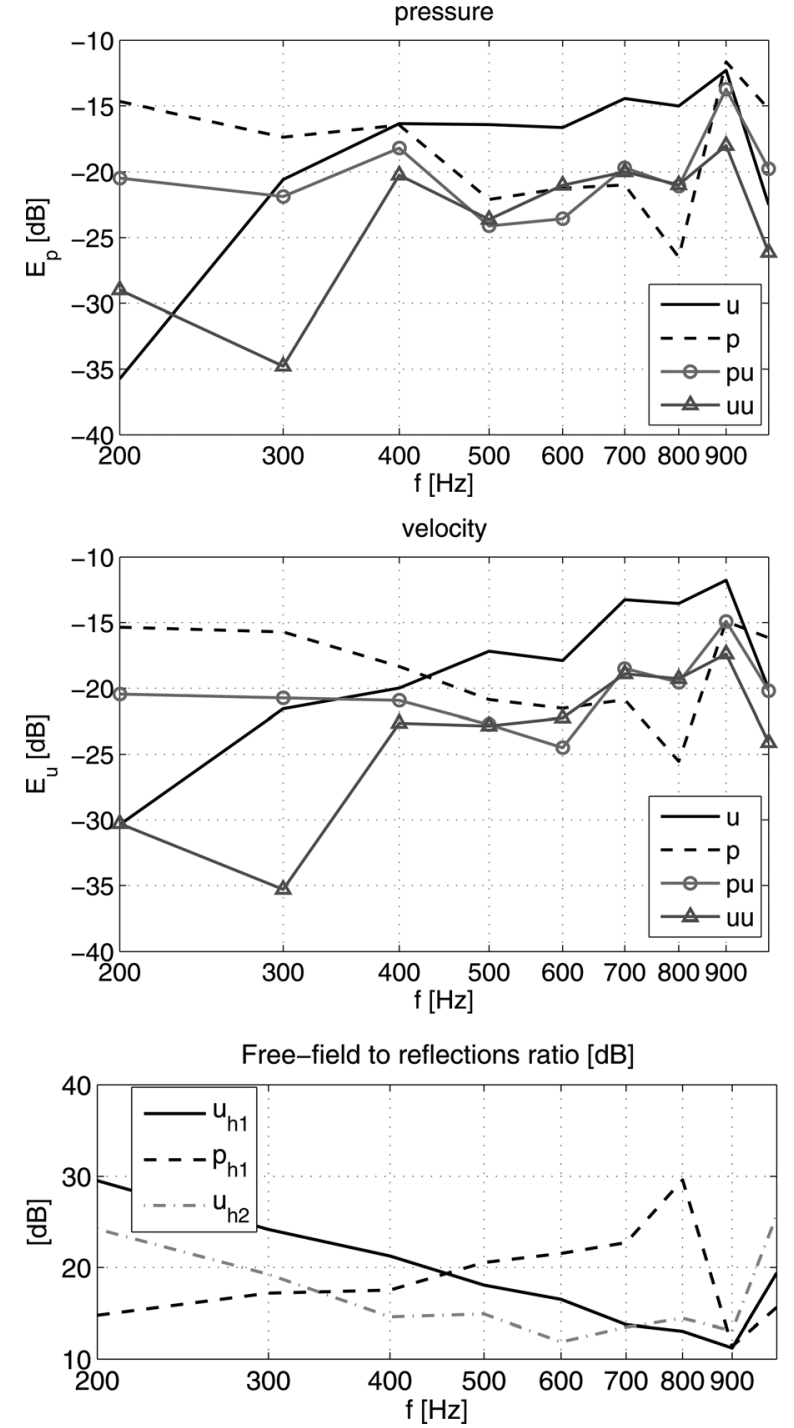

FIG. 5. Top: Error as in Eqs. (14) and (15) for a baffled plate radiating into a room. Bottom: Ratio between the pressure and velocity by the plate at $z_{h 1}$ and the reflected waves.

\section{EXPERIMENTAL STUDY}

An experimental study to examine the methods described in this paper was conducted. The measurements took place at the LVA, INSA-Lyon, France. The set-up consisted of a baffled plate radiating into a lightly damped room of dimensions $3.6 \times 2.15 \times 2 \mathrm{~m}^{3}$. The plate used was a $50 \times 70 \mathrm{~cm}^{2}$, $1 \mathrm{~mm}$ thick steel plate, driven near its center, at $(5,-10,0) \mathrm{cm}$. The sound pressure and the normal component of the particle velocity were measured with a line array of 11 particle velocity probes "Microflown p-u match," using a uniform interspacing of $6 \mathrm{~cm}$. The field was measured sequentially at $11 \times 16$ positions, over a total area of $40 \times 60 \mathrm{~cm}^{2}$. The measurement planes were $z_{h 1}=10 \mathrm{~cm}$ and $z_{h 2}=15 \mathrm{~cm}$, and $z_{h 1}$ served also as the reconstruction plane. The equivalent sources were retracted two inter-spacing distances from the reconstruction planes. The measurement set-up is shown in Fig. 7 and a picture of the measurement is shown in Fig. 8.

A 32 channel analyzer, OROS type OR38, was used. The plate was driven with random noise, and a force transducer at the driving point was used as a phase reference. The 


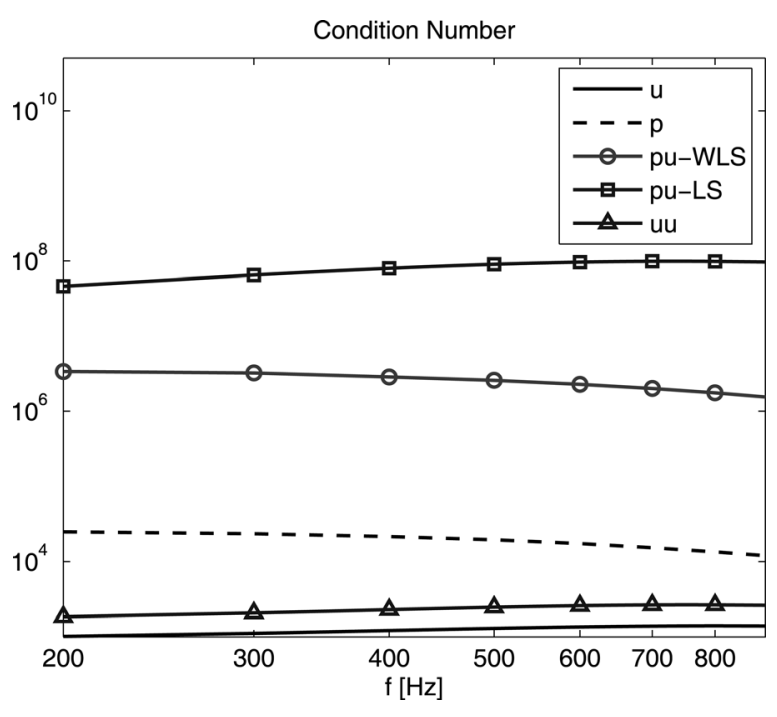

FIG. 6. Condition number (2-norm) of the matrices of the methods as used in this section. The least squares is also shown for comparison with the weighted least squares method.

spectral estimates were calculated with $0.33 \mathrm{~Hz}$ spectral resolution, corresponding to $3 \mathrm{~s}$ Hanning windows with $70 \%$ overlap, and 50 averages. The calibration of the probes was done by measuring at $20 \mathrm{~cm}$ from a monopole source in a anechoic room and calculating a correction for the probes to match the exact analytical ratio between pressure and velocity. ${ }^{25}$

In order to calculate the free field radiation from the plate, its vibration velocity was measured with a Polytec laser vibrometer OFV 056 over a grid of $26 \times 36$ positions, with $2 \mathrm{~cm}$ resolution. The free-field radiation was calculated using the wave superposition method, with the equivalent

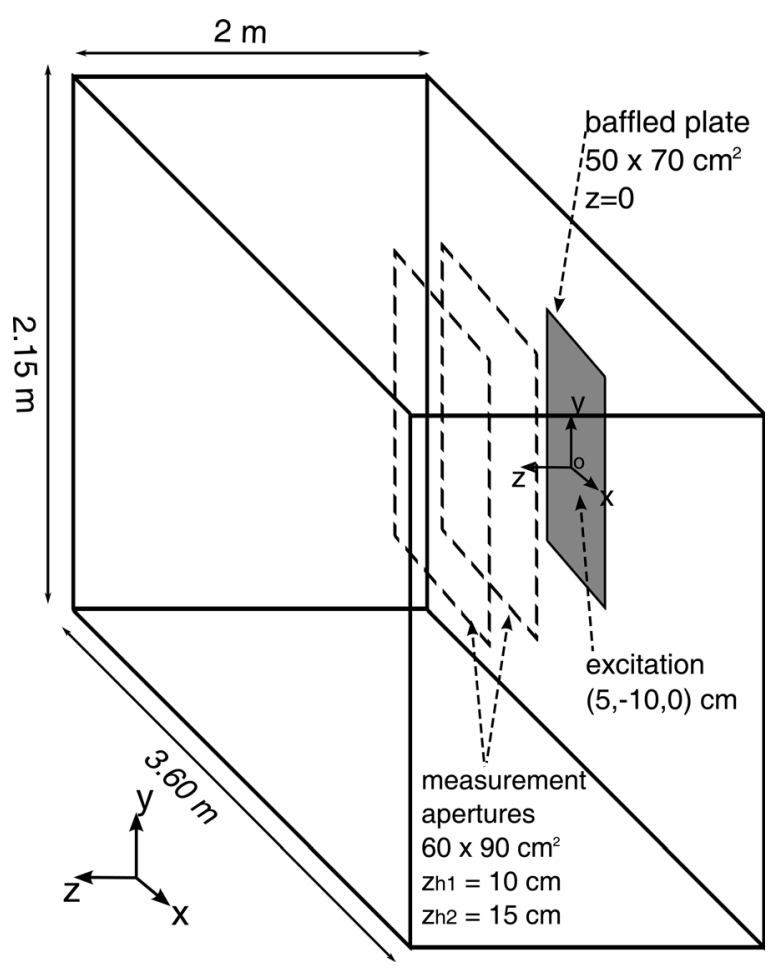

FIG. 7. Experimental set-up.
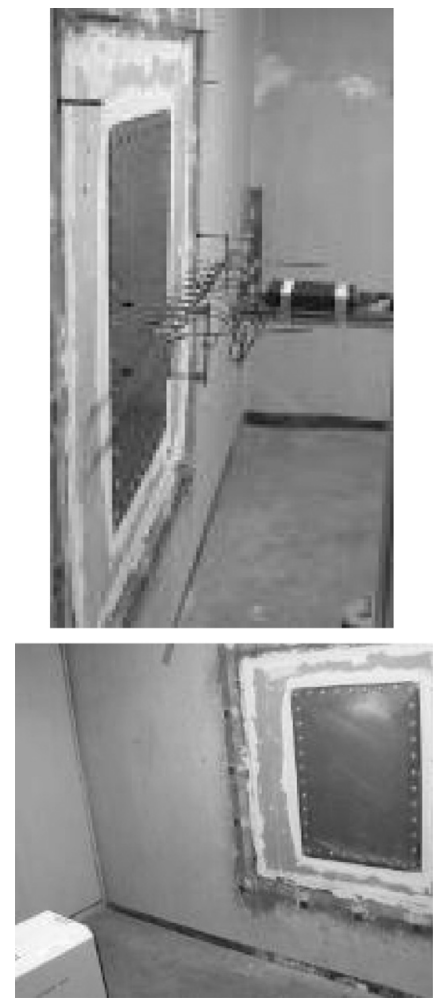

FIG. 8. Experimental measurement.

sources retracted $3 \mathrm{~cm}$ behind the plate. The results were identical to the ones obtained by evaluating numerically the Rayleigh integral. ${ }^{26}$ The resulting sound pressure and particle velocity served as the "true" reference fields for benchmarking.

Figure 9 illustrates the estimated sound pressure radiated from the source at $500 \mathrm{~Hz}$. It shows the true sound pressure calculated from the vibration of the plate, the direct reconstruction based on the sound pressure, particle velocity, double layer velocity ( $\mathrm{u}-\mathrm{u})$, and combined pressure-velocity (p-u) methods. The pressure based reconstruction is the least accurate. The $\mathrm{p}-\mathrm{u}$ method is better than the direct pressure reconstruction, although it seems that the velocity and $\mathrm{u}-\mathrm{u}$ reconstructions are the most accurate; the u-u method better recovers the deflection shape, but exhibits a large error around the low-left corner.

Figure 10 shows a comparison between the "true" freefield radiation of the plate and the estimation as a function of frequency. The frequencies shown correspond to the main natural frequencies of the plate, where the sound radiation is maximum and yields a better signal-to-noise ratio. The overall contribution of the reflections compared to the free-field radiation from the source was estimated to be of about $-10 \mathrm{~dB}$. It can be seen from Fig. 9 that the overall reconstruction error is notably high. This due to the reference "true" field used: Because it is not possible to measure "per $s e$ " the free-field radiation of the baffled plate with the p-u array, it is instead estimated based on the plate's vibration measured with a laser vibrometer. This introduces significant sources of error due to position bias, scattering by the array and preamplifier, calculation errors, etc. In spite of the high 

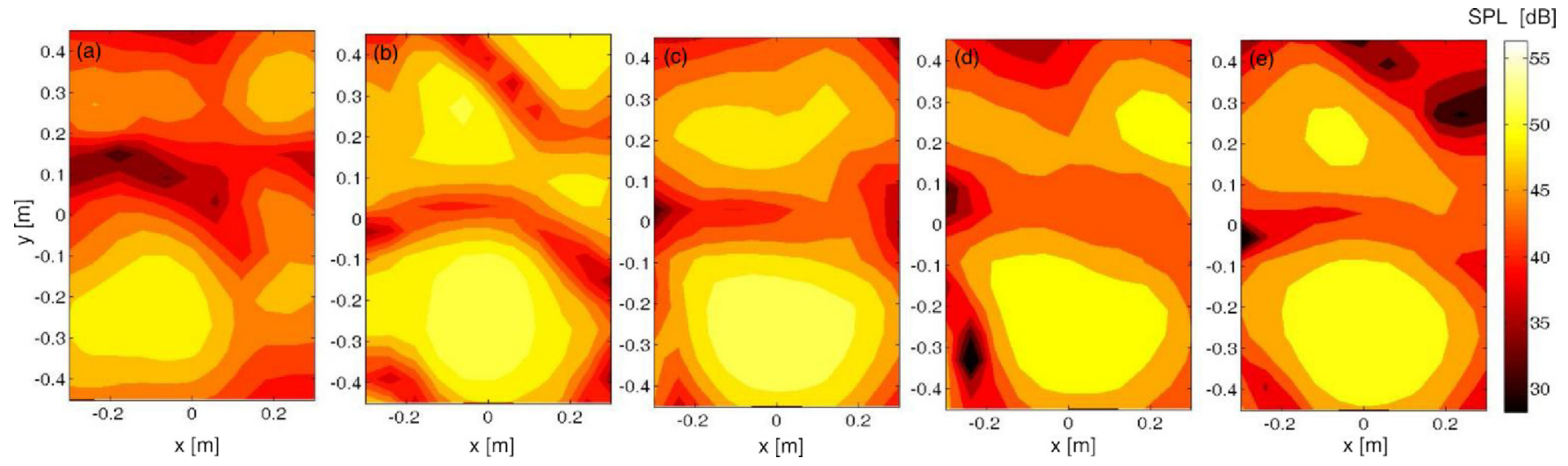

FIG. 9. (Color online) Radiation from the baffled plate at $500 \mathrm{~Hz}$. Sound pressure level in dB. (a) Free-field radiation; (b) direct reconstruction based on sound pressure measurements; (c) direct reconstruction based on normal velocity measurements; (d) reconstruction with the double layer velocity method; (e) reconstruction with the pressure-velocity method.

experimental error, the methods seem to follow some of the trends observed in the simulated results.

Note that at $700 \mathrm{~Hz}$ and above, the error increases presumably because of spatial aliasing due to the short wavelength of the evanescent waves (the flexural wavelength on the plate at $700 \mathrm{~Hz}$ is of about $12 \mathrm{~cm}$, whereas the transducer inter-spacing is $6 \mathrm{~cm}$ ). Although these aliased evanescent waves have decayed significantly at the measurement plane, they still can contribute to the error.

The results show that at low frequencies, the particle velocity based reconstruction and the two separation methods
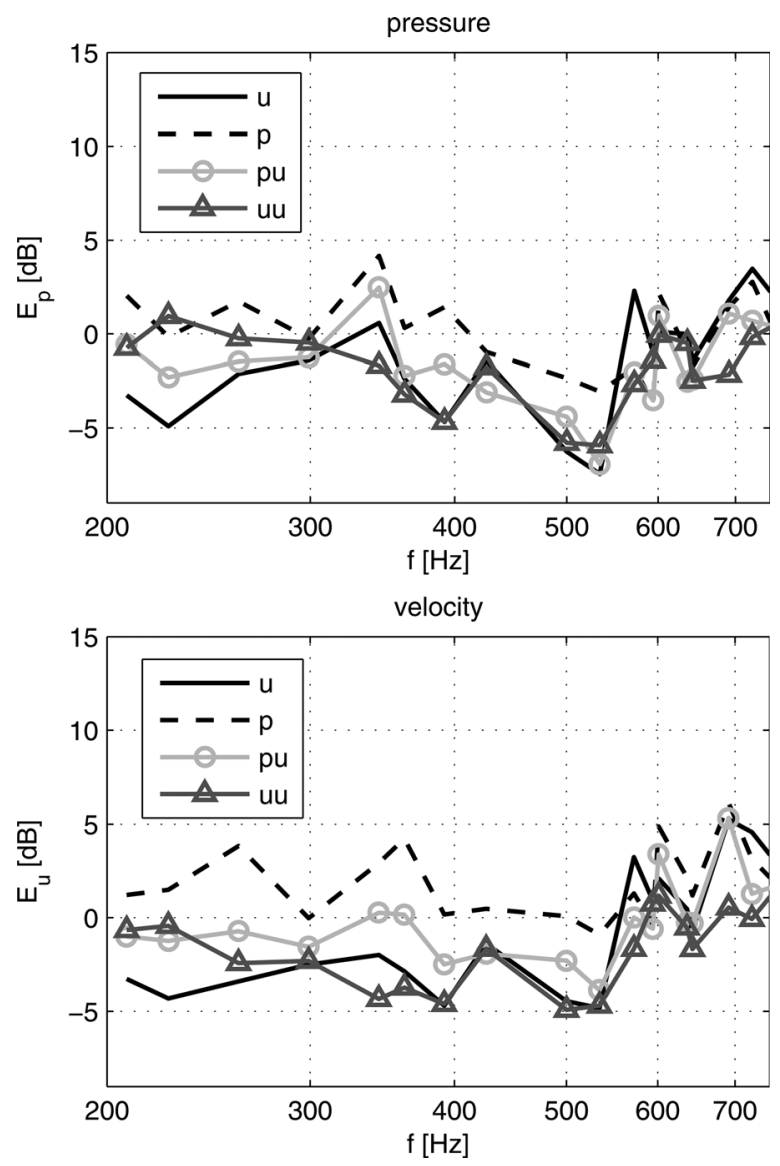

FIG. 10. Error as in Eqs. (14) and (15) for a baffled plate radiating into a lightly damped room. provide the best estimates. Particularly, at very low frequencies (below $300 \mathrm{~Hz}$ ) the direct velocity reconstruction is the most accurate, because the incoming sound vanishes at the plate's boundary. As the frequency increases, the direct single layer velocity reconstruction deteriorates due to the influence of the incoming sound, and becomes comparable to the separation methods. The accuracy of the two separation methods is comparable, even though below $300 \mathrm{~Hz}$, the results from the double layer velocity technique $(\mathrm{u}-\mathrm{u})$ are worse than expected. Nonetheless, on the whole, the $\mathrm{u}-\mathrm{u}$ method is somewhat more accurate than the $\mathrm{p}-\mathrm{u}$, and it is closer to the free-field radiation of the plate. A possible explanation for this is that, because of its directional characteristics, the normal component of the particle velocity is less influenced by flanking reflections from the floor, walls, and ceiling. Additionally, the $\mathrm{u}-\mathrm{u}$ method is more robust to background noise and measurement errors, and circumvents the so-called "p-u mismatch" inherent to the p-u method. ${ }^{12}$

\section{DISCUSSION}

It should be noted that the evaluation of the methods in this paper is based on a comparison between the free-field radiation by the source and the estimation by the separation methods, which merely separate sound coming from the two sides of the array (without compensating for the backscattered sound by the source, nor any other reflection coming from the source's side). Hence, the results evaluate how much the estimation corresponds to the free-field radiation of the source, but do not evaluate the accuracy of the separation as such. The study has shown that the over-all accuracy of the separation methods depends significantly on the magnitude of the disturbance of the measured field, pressure or particle velocity, resulting from the specific measurement situation.

Because the normal component of the particle velocity is directive, unlike the pressure, it is less affected by sound coming from the edges of the aperture. Furthermore, the normal component of incoming sound tends to vanish at the boundary of a rigid source. Therefore, when measuring close to the source, with a small stand-off distance relative to the wavelength $\left(z_{h}<0.1 \lambda_{z}\right)$, the direct reconstruction of the field based on the measurement of the particle velocity provides a robust and accurate estimate of the source's radiation. ${ }^{16}$ 
Nonetheless, measuring very close to the source also implies a potential risk of spatial aliasing, unless a sufficiently dense transducer array is used $(\Delta x<\lambda / 2)$. Consequently, the stand-off distance must be large enough so that the aliased evanescent waves have decayed at the measurement positions. When measuring at such stand-off distance the separation techniques can be useful. In this respect, the double layer velocity technique combines the properties of measuring the particle velocity (e.g., less truncation error, better conditioning to noise, less influence of flanking sound, etc. $)^{27}$ with the ability to distinguish sound from the two sides of the array; however, this comes at the expense of an additional layer at a greater stand-off distance, as opposed to the "more convenient" p-u method.

\section{CONCLUSIONS}

Two sound field separation methods based on the equivalent source method have been proposed and examined in this paper. The methods are based on the combined measurement of pressure and velocity, and on the measurement of the particle velocity in two parallel layers. Their performance in an enclosed space has been examined numerically and experimentally, and compared with the conventional direct single layer reconstructions based on pressure and velocity.

The results indicate that the direct reconstruction of the field based on particle velocity measurements is generally robust and can provide a fair estimation of the source's freefield radiation, particularly at low and mid frequencies near a rigid source, where the disturbance is minimal. At higher frequencies, separation techniques can be useful to minimize the influence of disturbing sound due to reflections. The accuracy of the proposed separation methods is comparable: On the one hand, the u-u method is less affected by flanking sound due to reflections and is more robust to measurement noise than the p-u method. On the other hand, due to the double layer configuration, the u-u method requires a larger back-propagation distance to the reconstruction surface than the p-u does. Nonetheless, if the level of disturbance is not critical, the direct reconstruction based on single layer measurements is certainly more accurate and convenient than using any of the sound-field separation techniques.

\section{ACKNOWLEDGMENTS}

The authors would like to thank Antonio A. Pereira for help with the experimental measurements and valuable discussion.

${ }^{1}$ J. D. Maynard, E. G. Williams, and Y. Lee, "Nearfield Acoustic Holography I: Theory of generalized holography and the development of NAH," J. Acoust. Soc. Am. 78, 1395-1413 (1985).

${ }^{2}$ E. G. Williams, Fourier Acoustics-Sound Radiation and Nearfield Acoustic Holography (Academic Press, San Diego, 1999), Chaps. 2-8.

${ }^{3}$ J. Bendat, "Solutions for the multiple input/output problem," J. Sound Vib. 44, 311-325 (1975).

${ }^{4}$ S. Price and R. Bernhard, "Virtual coherence: A digital signal processing technique for incoherent source identification," in Proceedings of the 4th
International Modal Analysis Conference 4, Los Angeles, CA (1986), Vol. II, pp. 1256-1262.

${ }^{5}$ J. Hald, "STSF-A unique technique for scan-based near-field acoustic holography without restrictions on coherence," Brüel \& Kjær Technical Review 1989-1 (1989). Available at www.bksv.com (last viewed 5/18/ 2012).

${ }^{6} \mathrm{M}$. A. Tomlinson, "Partial source discrimination in near field acoustic holography," Appl. Acoust. 57, 243-261 (1999).

${ }^{7}$ M. Tamura, "Spatial Fourier transform method of measuring reflection coefficients at oblique incidence. I: Theory and numerical examples," J. Acoust. Soc. Am. 88, 2259-2264 (1990).

${ }^{8} \mathrm{Z}$. Hu and J. S. Bolton, "The measurement of plane-wave reflection coefficients by using two-dimensional spatial transforms," J. Acoust. Soc. Am. 88, S173 (1990).

${ }^{9}$ J. Hald, "Patch holography in cabin environments using a two-layer hand held array with an extended SONAH algorithm," in Proceedings of Euronoise 2006, Tampere, Finland (Acoustical Society of Finland, Helsinki, 2006).

${ }^{10}$ C.-X. Bi, X.-Z. Chen, and J. Chen, "Sound field separation technique based on equivalent source method and its application in near field acoustic holography," J. Acoust. Soc. Am. 123, 1472-1478 (2008).

${ }^{11}$ N. P. Valdivia, E. G. Williams, and P. C. Herdic, "Approximations of inverse boundary element methods with partial measurements of the pressure field," J. Acoust. Soc. Am. 123, 109-120 (2008).

${ }^{12}$ F. Jacobsen and V. Jaud, "Statistically optimized near field acoustic holography using an array of pressure-velocity probes," J. Acoust. Soc. Am. 121, 1550-1558 (2007).

${ }^{13}$ C.-X. Bi and X.-Z. Chen, "Sound field separation technique based on equivalent source method using pressure-velocity measurements and its application in nearfield acoustic holography," in Proceedings of InterNoise 2008, Shangai, China (2008), paper No. IN08_0149.

${ }^{14}$ Y.-B. Zhang, F. Jacobsen, C.-X. Bi, and X.-Z. Chen, "Near field acoustic holography based on the equivalent source method and pressure-velocity transducers," J. Acoust. Soc. Am. 126, 1257-1263 (2009).

${ }^{15} \mathrm{C}$. Langrenne, M. Melon, and A. Garcia, "Measurement of confined acoustic sources using near-field acoustic holography," J. Acoust. Soc. Am. 126, 1250-1256 (2009).

${ }^{16} \mathrm{E}$. Fernandez-Grande and F. Jacobsen, "Sound field separation with a double layer velocity transducer array (L)," J. Acoust. Soc. Am. 130, 5-8 (2011).

${ }^{17}$ A. Sarkissian, "Method of superposition applied to patch near-field acoustic holography," J. Acoust. Soc. Am. 118, 671-678 (2005).

${ }^{18}$ G. H. Koopmann, L. Song, and J. B. Fahnline, "A method for computing acoustic fields based on the principle of wave superposition," J. Acoust. Soc. Am. 86, 2433-2438 (1989).

${ }^{19}$ D. Hallman and J. S. Bolton, "Nearfield acoustical holography for interior spaces," in Proceedings of Inter-Noise 94, Yokohama, Japan (1994), pp. 1879-1882.

${ }^{20}$ D. L. Hallman, J. S. Bolton, S. M. Dumbacher, and M. J. Lally, "Acoustic source location in vehicle cabins and free-field with nearfield acoustical holography via acoustic arrays," in Proceedings of the 19th International Seminar on Modal Analysis, Leuven, Belgium (1994), Vol. II, pp. 841-851.

${ }^{21}$ M. Villot, G. Chavériat, and J. Roland, "Phonoscopy: an acoustical holography technique for plane structures radiating in enclosed spaces," J. Acoust. Soc. Am. 91, 187-195 (1992).

${ }^{22} \mathrm{P}$. C. Hansen, "Analysis of discrete ill-posed problems by means of the L-curve," SIAM Rev. 34, 561-580 (1992).

${ }^{23}$ G. H. Golub and C. F. V. Loan, Matrix Computations (Johns Hopkins University Press, Baltimore, 1996), Chap. 5.

${ }^{24}$ A. A. Pereira and Q. Leclere, "Improving the equivalent source method for noise source identification in enclosed spaces," in 18 International Congress on Sound and Vibration, Rio de Janeiro, Brazil (2011), paper 2016.

${ }^{25} \mathrm{~F}$. Jacobsen and V. Jaud, "A note on the calibration of pressure-velocity sound intensity probes," J. Acoust. Soc. Am. 120, 830-837 (2006).

${ }^{26}$ E. G. Williams and J. D. Maynard, "Numerical evaluation of the Rayleigh integral for planar radiators using the FFT," J. Acoust. Soc. Am. 72, 2020-2030 (1982).

${ }^{27}$ F. Jacobsen and Y. Liu, "Near field acoustic holography with particle velocity transducers," J. Acoust. Soc. Am. 118, 3139-3144 (2005). 